\title{
Exploring the Mechanism of Quercetin for the Treatment of Atrial Fibrillation by Geo Gene Chip Combined With Network Pharmacology and Molecular Docking
}

$\tan x$ in

First Affiliated Hospital of Bengbu Medical College https://orcid.org/0000-0002-6576-0401

Wei Xian

The First Affiliated Hospital of Bengbu Medical College

Xiaorong Li

The First Affiliated Hospital of Bengbu Medical College

\section{Yongfeng Chen}

The First Affiliated Hospital of Bengbu Medical College

Jiayi Geng

Bengbu Medical College

Qiyi Wang

Bengbu Medical College

Qin Gao

Bengbu Medical College

\section{Bi Tang}

The First Affiliated Hospital of Bengbu Medical College

\section{Hongju Wang}

The First Affiliated Hospital of Bengbu Medical College

Pinfang Kang ( $\sim$ kangpinfang.1016@163.com )

The First Affiliated Hospital of Bengbu Medical College https://orcid.org/0000-0001-7084-7862

\section{Research Article}

Keywords: atrial fibrillation, quercetin, pharmacological analysis, molecular docking technology, inflammation response

Posted Date: November 23rd, 2021

DOI: https://doi.org/10.21203/rs.3.rs-1091154/v1 
License: (c) (i) This work is licensed under a Creative Commons Attribution 4.0 International License. Read Full License 


\section{Abstract}

\section{Purpose}

Atrial fibrillation (AF) is a common atrial arrhythmia. Quercetin (Que) has some advantages in the treatment of cardiovascular disease arrhythmias, but its specific drug mechanism of action needs further investigation. To explore the mechanism of action of Que in AF, core target speculation and analysis were performed using network pharmacology and molecular docking methods.

Methods

Que chemical structures were obtained from Pubchem. TCMSP, Swiss Target Prediction, Drugbank, STITCH, Binding DB, Pharmmapper, CTD, GeneCards, DISGENET and TTD were used to obtain drug component targets and AF-related genes, and extract AF from normal tissues by GEO database differentially expressed genes. Then, the intersecting genes were obtained by online Wayne mapping tool. The intersection genes were introduced into the top five targets selected for molecular docking via protein-protein interaction (PPI) network to verify the binding activity between Que and the target proteins. GO and KEGG enrichment analysis of the intersected genes using program $\mathrm{R}$ was performed to further screen for key genes and key pathways.

Results

There were 65 effective targets for Que and AF. Through further screening, the top 5 targets were IL6, VEGFA, JUN, MMP9 and EGFR. Que treatment of AF may involve signaling pathways such as lipid and atherosclerosis pathway, AGE-RAGE signaling pathway in diabetic complications, MAPK signaling pathway and IL-17 signaling pathway. Molecular docking suggests that Que has strong binding to key targets.

\section{Conclusion}

This study systematically elucidates the key targets of Que treatment for AF and the specific mechanisms through network pharmacology as well as molecular docking, providing a new direction for further basic experimental exploration and clinical treatment.

\section{Introduction}

Atrial fibrillation (AF) is by far the most common type of atrial arrhythmia and is a risk factor for increased cardiovascular mortality[1]. The prevalence of AF increases with age, doubling every 10 years after age 50 years and reaching $10 \%$ in patients $\geq 80$ years. Furthermore, the number of patients with AF is expected to increase significantly in the coming decades[2,3]. Oral anticoagulant drugs are currently one of the main treatment modalities for AF, such as rivaroxaban and dabigatran[4, 5]. In addition, relevant studies have reported that AF patients will increase significantly in the next few decades. 
Therefore, exploring new drugs to reduce the danger of bleeding in patients with $A F$, improve the cardiac function of patients and the complications of $\mathrm{AF}$ requires further research.

Quercetin (Que) is widely found in vegetables and fruits, and Que is also found in several herbal medicines. The therapeutic potential of Que has been widely studied because of its ability to inhibit lowdensity lipoprotein (LDL) oxidation, reduce adhesion molecules and other inflammatory markers, prevent neuronal oxidation and inflammatory damage, and platelet anti-aggregation[6, 7]. Que has been reported to show significant therapeutic effects in animal models of heart failure, arrhythmias and hypertension in various cardiovascular diseases[8-11]. Recent studies have found that in a rat model of arrhythmia after myocardial ischemia and reperfusion injury, the administration of Que significantly reduced the time to arrhythmia as well as lactate dehydrogenase (LDH) levels of myocardial injury[12]. Based on these comprehensive data, Que is one of the new therapeutic approaches for the prevention of cardiovascular diseases, but there is still lack of the related research in vitro and in vivo as well as clinical studies of Que for AF treatment, so we think it is necessary to do the systematic and in-depth studies on the molecular mechanisms of Que for AF treatment.

Network pharmacology is to further analyze the relationship between drugs, diseases and targets by collecting various database data, data analysis and simulation[13]. Therefore, by using a network pharmacological analysis method, we investigated the targets of action in Que for the treatment of AF and analyzed their relevant target biological pathways, which laid a good foundation for further in-depth study of the mechanism of action of Que for the treatment of AF. The detail flow chart of this study is shown in Figure 1.

\section{Materials And Methods}

\section{Acquisition of Que targets}

Using the TCMSP (http://tcmspw.com/)[14], PharmMapper (http://www.lilab-ecust.cn/pharmmapper/) [15], SwissTargetPrediction (http://www.swisstargetprediction.ch/)[16], Drugbank (https://go.drugbank.com/)[17], Binding database (http://www.bindingdb.org/bind/index.jsp)[18], STITCH (http://stitch.embl.de/ cgi/input.pl?Userld=MxcokIPgQVMy\&sessionld=032YjbzG5Gio) and other databases to obtain Que targets, default HumanProtein Targets Only (Retrieved on:2021.7.10).

\section{Acquisition of $\mathrm{AF}$ related targets}

The CTD (http://ctdbase.org)[19], GeneCards (https://www.genecards.org/)[20], DISGENET(https://www.disgenet.org/)[21], Therapeutic Target Database (http://db.idrblab.net/ttd/)[22], were used to "Atrial fibrillation" was used as a keyword to search for the relevant genes. The collected targets were combined and eliminated by the median algorithm to obtain disease targets. GSE31821 data were imported into the online analysis tool GEO2R for processing to obtain differentially expressed genes. The gene screening criteria were $\mathrm{P}<0.05$ and $|\log \mathrm{FC}| \geq 0.5$ to obtain differentially expressed genes 
between normal tissues and $\mathrm{AF}$, and further volcano maps of differentially expressed genes were drawn[23].

\section{Cross-tabulation of drugs, diseases and GEO}

By using the online mapping tool (http://www.ehbio.com/test/venn/\#/), intersections of drug targets, disease targets and differential genes can be obtained.

\section{Construction of protein-protein interaction network (PPI)}

The intersecting genes were imported into the String database [24] (https://string-db.org/cgi/input.pl) to construct the protein-protein interaction network (PPI), and the species selectors, exported in tsv format. The tsv was imported into Cytoscape 3.7.2 software (http://www.cytoscape.org/) for adjustment as well as to obtain key targets using the cytoHubba plugin. Simultaneous protein functional classification of the intersecting targets using the Panther database.

\section{GO analysis and KEGG pathway and Que-primary pathway-AF network construction}

To further understand the function and role in signaling pathways of the intersecting genes, the gene abbreviations (Symbol) were transformed into gene ID (EntrezID), and the results were analyzed visually by the $\mathrm{R}$ language package[6], as well as the gene ontology (GO) of the above intersecting genes, GO analysis can further explain the role of the intersecting genes in molecular function (MF), biological process (BP) and cellular component (CC) of AF resistance. The Kyoto encyclopedia of genes and genomes (KEGG) enrichment analysis was used to investigate the major anti-AF signaling pathways involved in the crossover genes. The core target network of "Que-major pathway-AF" was also constructed.

\section{Molecular Docking and differential analysis of core targets}

The known key targets were molecularly docked to Que to obtain docking affinities reflecting their stability. The PDB database was used to download the core protein molecular structures and PUBCHEM to download the structures of Que. The protein and Que structures were imported into Auto Dock Tools software for processing and molecular docking, and intermolecular docking affinities were calculated and PyMol 2.4.0 was used for visualization[25,26]. Changes in core targets were analyzed using differential expression between the normal and AF groups from the GEO database. GraphPad Prism software was used for graphical visualization. Values are expressed as mean \pm SD.

\section{Results}

\section{Information on potential targets of Que and AF}

The chemical structure of quercetin is shown in Figure 2A. Que potential targets were obtained by TCMSP, PharmMapper, Swiss Target Prediction, Drugbank, Stitch and Binding database databases, and a total of 
449 were obtained by screening de-duplication and integration. A total of 2201 disease targets were screened using CTD, GeneCards, Disgenet and TTD databases, and 3096 differential genes between normal and AF patients were obtained using the GEO database, of which 462 were upregulated and 2634 were downregulated. From the volcano plot (Figure 2B) and the difference plot (Figure 2C), it can be seen that there are significant genetic differences between normal human atrial tissues and atrial tissues from AF patients. Combining drug targets, disease targets and GEO targets, 65 core targets, including IL-6, EGFR, MMP9, ESR1 (etc) were obtained by online mapping of Venn diagram (Figure 2D).

\section{PPI network and Key Targets of Que for AF}

The Que and AF intersection targets were imported into String database, species limited to human, and the protein interactions network was obtained, which was further imported into Cytoscape for topological analysis, and the results suggested that there were 65 nodes and 575 edges in the network, and the key core targets were obtained using cytoHubba plugin (Figure 2E), ranking the top 10 key targets for treating AF targets IL-6, VEGFA, JUN, MMP9, EGFR, CCND1, CXCL8, PTGS2, ESR1 and MYC, and the detailed information of the top 10 targets ranked by the key target Degree value is shown in Table 1.

\section{GO function analysis and classification}

To further clarify the mechanism of action of quercetin in the treatment of AF, we classified 65 core targets into proteins using the PANTHER database (Figure 3A), found that they were mainly focused on protein modifying enzymes (20.4\%), gene-specific transcriptional regulators (18.40\%), and metabolite interconverting enzymes (18.40\%). 65 core genes were introduced into the R package, and the $\mathrm{GO}$ functional results (Figure 3B), the biological process (BP) was mainly focused on: response to lipopolysaccharide, response to molecule of bacterial origin, regulation of inflammatory response, response to oxidative stress, and epithelial cell proliferation; cell composition (CC) is mainly focused on: vesicle lumen, membrane raft, membrane microdomain, membrane region, focal adhesion; molecular functions (MF) mainly focus on: DNA-binding transcription factor binding, RNA polymerase II-specific DNA-binding transcription factor binding, cytokine receptor binding, monocarboxylic acid binding, and growth factor binding. In addition, we used the MCODE tool to identify highly interconnected clusters in the PPI network, and the MCODE clusters were classified into three types, including regulation of smooth muscle cell proliferation (Figure 3C), positive regulation of protein import into nucleus (Figure 3D), negative regulation of intracellular signal transduction (Figure 3E).

\section{KEGG pathway analysis and core pathway network construction}

The pathways of action were clarified by KEGG enrichment analysis of 65 intersecting genes, with 157 enrichment terms, of which the top 30 most significant pathways (Figure 4A), the core targets may affect the lipid and atherosclerosis, AGE-RAGE signaling pathway in diabetic complications, MAPK signaling pathway, and IL-17 signaling pathway, suggesting that Que may be effective in treating AF by modulating the above signaling pathways. 
The top 10 relevant pathways of Que for AF were screened (Table 2) and imported into Cytoscape to construct the "Que-Major Pathways-AF" network (Figure 4B). After screening out the KEGG-enriched pathways that were not related to AF disease, it was found that the important pathway in Que for AF treatment was probably related to MAPK signaling pathway, and the detailed pathway map was obtained by $\mathrm{R}$ package analysis (Figure $4 \mathrm{C}$ ), in which the red area was signal upregulation.

\section{Molecular docking and differential analysis of core targets}

The five core targets with high Degree values were selected for molecular docking to verify the binding activity of Que active ingredients to potential anti-AF targets. The average docking affinity of the results was $-5.042 \mathrm{kcal} / \mathrm{mol}$, as detailed in Table 3, where the larger the absolute value of the docking affinity, the more stable the binding proved to be, indicating that Que has strong binding to the core targets. The detailed diagram of docking with each key target site is shown in Figure 5.

Using the difference data of control group and AF group in GEO database, the differential expression of the first five core targets in Que treatment of AF diseases (Figure 6). VEGFA was significantly upregulated, IL-6, JUN and VEGFA were significantly downregulated compared to controls, while MMP9 targets were not statistically significant, which was considered to be related to the small sample size. The above results suggest that the core targets are closely related to the pathological changes of AF.

\section{Discussion}

One study found that the increased risk of cardiovascular disease in some populations was associated with low dietary intake of flavonoids, including Que[27]. It has been found that Que played the antiischemic and anti-arrhythmic effects in clinical studies[9-11], it leads to great interest and concern of scientists, but the specific mechanisms of Que in the treatment of AF remains unclear. In this study, we investigated the mechanism of Que in the treatment of AF based on network pharmacology analysis. In this study, 449 Que potential targets and 2201 AF targets were obtained from each database respectively, 3096 AF differential genes were obtained from the GEO database. The intersection of the 3 was taken to obtain 65 core genes for Que treatment of AF. The above core genes were screened for the top 5 hub target genes of IL-6, VEGFA, JUN, MMP9 and EGFR by Degree value. GO analysis confirmed that Que had the potential to play an important role in the pathogenesis of AF through multiple biological pathways, and KEGG analysis obtained key pathways of Que for AF, mainly involving lipid and atherosclerosis pathways, the AGE-RAGE signaling pathwayin diabetic complications, MAPK signaling pathway and IL-17 signaling pathway, etc. Through further screening, we found that MAPK pathway plays a key role in the treatment of $\mathrm{AF}$.

IL-6 plays a key role in immune, tissue regeneration and metabolism. It was shown that IL-6 in the left atrium was found to promote early atrial fibrosis through the pSTAT3/STAT3 signaling pathway by establishing postoperative AF mice, and similarly higher IL-6 concentrations were found in the pericardial drainage fluid of patients with postoperative AF in a prospective clinical study[28]. Wu reported inhibiting IL-6 inflammatory factor release reduced early atrial fibrosis and its duration in SD rats with postoperative 
AF[29]. Studies based on clinical follow-up investigations have found that measurement of IL-6 concentrations could provide an independent prognostic signal as well as a predictor of recurrence for mortality in AF patients[30,31]. Therefore, IL-6 has the potential to be a therapeutic target for AF. The next key gene VEGFA is a key factor in the growth process of blood vessels and endothelial cells. Its main function is to induce endothelial cell proliferation and inhibit apoptosis[32]. It is well known that AF can lead to endothelial dysfunction[33], and Wang reported that the plasma levels of VEGFA were significantly elevated in AF patients[34], suggesting that endothelial dysfunction may be one of the etiologies of AF, VEGFA may be a potential therapeutic target for AF.

The next gene is Transcription factor JUN, It has been shown that the transcription factor complex AP-1 plays a major role in cardiac hypertrophy, and knockout of c-jun pure mice develops in a process leading to cardiac malformations[35], activation of c-jun impaired cell-cell communication between atrial myocytes and ultimately promotes the development of atrial arrhythmias[36, 37]. Therefore, JUN has the potential to be a therapeutic target for AF. The next gene MMP9 is a matrix metalloproteinase that plays an important role in local protein hydrolysis, cell migration, apoptosis and signaling in the extracellular matrix. During AF development, overproduction of reactive oxygen species activated MAPK signaling pathway further increasing MMP9 expression[38]. Moe GW reported inhibiting MMP9 expression in canine AF model improved cardiomyocyte hypertrophy and reduced myocardial fibrosis, as well as reduced AF duration[39]. Recent clinical studies have found that the plasma MMP9 level in AF patients were higher than that in controls, which suggested MMP9 could be as a target for the treatment of AF[30, 40]. The last of the first five core genes is EGFR, whose main function is to regulate cell growth, proliferation and apoptosis, and is a key regulator of cardiac organogenesis and adult heart[41]. In AF fibrotic atrial tissues, EGFR expression was increased, probably due to the cleavage by pro-fibrotic metalloproteinases during atrial remodeling[42]. The plasma level of EGFR in clinical AF patients was significantly higher than that in the control group[43], These results suggest that EGFR plays an important role in AF. Overall, the top five core genes were found to be supported by previous studies based on network pharmacology studies.

The results of KEGG enrichment analysis suggested that the lipid and atherosclerosis pathway, AGERAGE signaling pathway in diabetic complications, MAPK signaling pathway and IL-17 signaling pathway played the major roles in the treatment of AF with Que. One of the main causative factors of atherosclerosis and cardiovascular disease is dyslipidemia, which is considered one of the major risk factors for AF, and several clinical studies had demonstrated that LDL and total cholesterol were negatively correlated with the occurrence of AF[44-46]. It is suggested that lipids and atherosclerosis may be involved in the pathogenesis of AF. The AGE-RAGE signaling pathway is an important link in the development of diabetic disease, and this signaling pathway directly mediates diabetic vascular calcification and vasoconstriction-diastolic dysfunction, and a meta-analysis showed that type 2 diabetes increased the risk of AF by $40 \%[47]$. Recent studies had found that the AGE-RAGE signaling pathway was associated with the development of AF[48, 49], and that when AGE bind to RAGE, it produced inflammatory cytokines and cell adhesion molecules that further induced structural and electrical remodeling of the atria. Raposeiras-Roubín found significantly higher AGE and RAGE plasma levels in AF 
patients than in normal subjects, and several prospective studies have also shown that RAGE predicts future cardiovascular events and death in diabetic patients[50]. This suggesting that this pathway may mediate the progression of AF.

MAPK signaling pathway plays an important role in cell growth, proliferation and fibroblast growth. Li found that atrial structural remodeling in an AF mouse model could be attenuated by decreasing MAPK expression[51]. It has been demonstrated that activation of phosphorylated MAPK significantly increases susceptibility to AF[52], in detail MAPK pathway (Figure 4C), red upregulated targets get inflammation and apoptosis play an important role in the development of AF disease. Inflammation is one of the important pathogenic mechanisms of $A F$, and there is an increasing evidence supporting the involvement of inflammation and immune response in the development and progression of AF. Many studies have found that IL-17 signaling pathway stimulates the release of pro-inflammatory cytokines, which promote myocardial fibrosis and thus induce the development of AF[53, 54]. In addition, IL-17 promotes AF development in rats model with aseptic pericarditis by promoting cardiac inflammatory expression and atrial fibrosis.[53,55] Therefore, the results of the present study suggest that these four signaling pathways may be involved in the mechanism of Que treatment of AF.

GO analysis revealed the biological processes mainly focused on responses to lipopolysaccharide, regulation of inflammatory responses, phosphatidylinositol-mediated signaling, responses to oxidative stress and responses to epithelial cell proliferation related to the aforementioned pathway-mediated biological processes, which are also consistent with the aforementioned pathway-mediated biological processes. Molecular docking also suggested strong binding of Que to the above key targets and also validated the therapeutic effect of Que on AF targets at the molecular level.

\section{Conclusions}

In summary, we have clarified the key target of Que treatment of AF through network pharmacology, obtained the core signaling pathways for the treatment of AF through GO and KEGG analysis and screening, and validated the binding of Que to the key targets using molecular docking technology. However, the main limitation of this study is the lack of drug intervention in AF animal model experiments and verification of key pathways. Therefore, this study can provide a basis for further research in basic experiments, as well as new ideas and methods for drug development.

\section{Declarations}

Author Contributions (I) Conception and design: Xin Tan, Pinfang Kang; (II) Administrative support: Qin Gao, HongJu Wang, PinFang Kang, QiYi Wang; (III) Collection and assembly of data: Wei Xian, Xiaorong Li, Yongfeng Chen, Jiayi Geng; (IV) Data analysis and interpretation: Tan Xin, Wei Xian; (V) Manuscript writing: Tan Xin, Pinfang Kang. 
Funding This study was supported by the National Science Foundation of China (Grant No.81970313), Anhui Provincial Science and Technology Tackling Project (1804h08020246), Natural Science Foundation of Anhui Province Project (1908085QH353), Key Program of Anhui Educational Committee (KJ2020A0560), Anhui Provincial University Excellent Talent Fund (gxyq2020022), Bengbu Medical College 512 Excellent Talent Fund (by41201315), Bengbu Medical College Graduate Innovation Program (Byycx20025, Byycx20026).

Data Availability Author can confirm that all relevant data are included in the article and/or its supplementary information files.

\section{Declarations}

Ethical Approval This article does not contain any studies with human participants or animals performed by any of the authors.

Informed Consent This article does not contain any studies with human participants performed by any of the authors.

Consent for publication All authors agree to public.

Conflict of Interest The authors declare that there is no conflict of interest.

\section{References}

1. Katritsis, D.G., Is atrial fibrillation an inflammatory disorder? Eur Heart J, 2006. 27(7): p. 886; author reply 886 .

2. Go, A.S., E.M. Hylek, K.A. Phillips, et al., Prevalence of diagnosed atrial fibrillation in adults: national implications for rhythm management and stroke prevention: the AnTicoagulation and Risk Factors in Atrial Fibrillation (ATRIA) Study. Jama, 2001. 285(18): p. 2370-5.

3. Kannel, W.B., P.A. Wolf, E.J. Benjamin, and D. Levy, Prevalence, incidence, prognosis, and predisposing conditions for atrial fibrillation: population-based estimates. Am J Cardiol, 1998. 82(8a): p. $2 \mathrm{n}-9 \mathrm{n}$.

4. Writing Group, M., D. Mozaffarian, E.J. Benjamin, et al., Heart Disease and Stroke Statistics-2016 Update: A Report From the American Heart Association. Circulation, 2016. 133(4): p. e38-360.

5. Tomaselli, G.F., K.W. Mahaffey, A. Cuker, et al., 2020 ACC Expert Consensus Decision Pathway on Management of Bleeding in Patients on Oral Anticoagulants: A Report of the American College of Cardiology Solution Set Oversight Committee. J Am Coll Cardiol, 2020. 76(5): p. 594-622.

6. Torres, N., J. Martinez-Luscher, E. Porte, R. Yu, and S. Kaan Kurtural, Impacts of leaf removal and shoot thinning on cumulative daily light intensity and thermal time and their cascading effects of 
grapevine (Vitis vinifera L.) berry and wine chemistry in warm climates. Food Chem, 2021. 343: p. 128447.

7. Flores, I.R., M.S. Vasquez-Murrieta, M.O. Franco-Hernandez, et al., Bioactive compounds in tomato (Solanum lycopersicum) variety saladette and their relationship with soil mineral content. Food Chem, 2021. 344: p. 128608.

8. Sharma, A., M. Parikh, H. Shah, and T. Gandhi, Modulation of Nrf2 by quercetin in doxorubicintreated rats. Heliyon, 2020. 6(4): p. e03803.

9. Patel, R.V., B.M. Mistry, S.K. Shinde, et al., Therapeutic potential of quercetin as a cardiovascular agent. Eur J Med Chem, 2018. 155: p. 889-904.

10. Malishevskaia, I.V., T.A. Ilashchuk, and I.V. Okipniak, [Therapeutic efficacy of quercetin in patients with is ischemic heart disease with underlying metabolic syndrome]. Georgian Med News, 2013(225): p. 67-71.

11. Chekalina, N.I., S.V. Shut, T.A. Trybrat, et al., Effect of quercetin on parameters of central hemodynamics and myocardial ischemia in patients with stable coronary heart disease. Wiad Lek, 2017. 70(4): p. 707-711.

12. Lu, J., Y. Meng, R. Wang, and R. Zhang, Anti-arrhythmogenic effects of quercetin postconditioning in myocardial ischemia/reperfusion injury in a rat model. Journal of King Saud University - Science, 2020. 32(3): p. 1910-1916.

13. Silverman, E.K., H. Schmidt, E. Anastasiadou, et al., Molecular networks in Network Medicine: Development and applications. Wiley Interdiscip Rev Syst Biol Med, 2020. 12(6): p. e1489.

14. Ru, J., P. Li, J. Wang, et al., TCMSP: a database of systems pharmacology for drug discovery from herbal medicines. J Cheminform, 2014. 6: p. 13.

15. Wang, X., Y. Shen, S. Wang, et al., PharmMapper 2017 update: a web server for potential drug target identification with a comprehensive target pharmacophore database. Nucleic Acids Res, 2017. 45(W1): p. W356-W360.

16. Daina, A., O. Michielin, and V. Zoete, SwissTargetPrediction: updated data and new features for efficient prediction of protein targets of small molecules. Nucleic Acids Res, 2019. 47(W1): p. W357W364.

17. Wishart, D.S., Y.D. Feunang, A.C. Guo, et al., DrugBank 5.0: a major update to the DrugBank database for 2018. Nucleic Acids Res, 2018. 46(D1): p. D1074-d1082.

18. Gilson, M.K., T. Liu, M. Baitaluk, et al., BindingDB in 2015: A public database for medicinal chemistry, computational chemistry and systems pharmacology. Nucleic Acids Res, 2016. 44(D1): p. 
D1045-53.

19. Davis, A.P., C.J. Grondin, R.J. Johnson, et al., The Comparative Toxicogenomics Database: update 2017. Nucleic Acids Res, 2017. 45(D1): p. D972-d978.

20. Rebhan, M., V. Chalifa-Caspi, J. Prilusky, and D. Lancet, GeneCards: integrating information about genes, proteins and diseases. Trends Genet, 1997. 13(4): p. 163.

21. Zhang, W., A. Bojorquez-Gomez, D.O. Velez, et al., A global transcriptional network connecting noncoding mutations to changes in tumor gene expression. Nat Genet, 2018. 50(4): p. 613-620.

22. Wang, Y., S. Zhang, F. Li, et al., Therapeutic target database 2020: enriched resource for facilitating research and early development of targeted therapeutics. Nucleic Acids Res, 2020. 48(D1): p. D1031d1041.

23. Yu, S., Q. Guo, T. Jia, et al., Mechanism of Action of Nicotiflorin from Tricyrtis maculata in the Treatment of Acute Myocardial Infarction: From Network Pharmacology to Experimental Pharmacology. Drug Des Devel Ther, 2021. 15: p. 2179-2191.

24. Szklarczyk, D., A. Franceschini, S. Wyder, et al., STRING v10: protein-protein interaction networks, integrated over the tree of life. Nucleic Acids Res, 2015. 43(Database issue): p. D447-52.

25. Morris, G.M., R. Huey, W. Lindstrom, et al., AutoDock4 and AutoDockTools4: Automated docking with selective receptor flexibility. J Comput Chem, 2009. 30(16): p. 2785-91.

26. Seeliger, D. and B.L. de Groot, Ligand docking and binding site analysis with PyMOL and Autodock/Vina. J Comput Aided Mol Des, 2010. 24(5): p. 417-22.

27. Knekt, P., R. Jarvinen, A. Reunanen, and J. Maatela, Flavonoid intake and coronary mortality in Finland: a cohort study. Bmj, 1996. 312(7029): p. 478-81.

28. Liu, Y., F. Wu, Y. Wu, et al., Mechanism of IL-6-related spontaneous atrial fibrillation after coronary artery grafting surgery: IL-6 knockout mouse study and human observation. Transl Res, 2021. 233: p. 1631.

29. Tsioufis, C., D. Konstantinidis, I. Nikolakopoulos, et al., Biomarkers of Atrial Fibrillation in Hypertension. Curr Med Chem, 2019. 26(5): p. 888-897.

30. Aulin, J., Z. Hijazi, A. Siegbahn, et al., Serial measurement of interleukin- 6 and risk of mortality in anticoagulated patients with atrial fibrillation: Insights from ARISTOTLE and RE-LY trials. J Thromb Haemost, 2020. 18(9): p. 2287-2295.

31. Aulin, J., A. Siegbahn, Z. Hijazi, et al., Interleukin-6 and C-reactive protein and risk for death and cardiovascular events in patients with atrial fibrillation. Am Heart J, 2015. 170(6): p. 1151-60. 
32. Tammela, T., G. Zarkada, E. Wallgard, et al., Blocking VEGFR-3 suppresses angiogenic sprouting and vascular network formation. Nature, 2008. 454(7204): p. 656-60.

33. Takahashi, N., Y. Ishibashi, T. Shimada, et al., Atrial fibrillation impairs endothelial function of forearm vessels in humans. J Card Fail, 2001. 7(1): p. 45-54.

34. Wang, K., Y. Liu, S. Huang, et al., Does an imbalance in circulating vascular endothelial growth factors (VEGFs) cause atrial fibrillation in patients with valvular heart disease? J Thorac Dis, 2019. 11(12): p. 5509-5516.

35. Passegué, E., W. Jochum, A. Behrens, R. Ricci, and E.F. Wagner, JunB can substitute for Jun in mouse development and cell proliferation. Nat Genet, 2002. 30(2): p. 158-66.

36. Yan, J., W. Kong, Q. Zhang, et al., c-Jun N-terminal kinase activation contributes to reduced connexin43 and development of atrial arrhythmias. Cardiovasc Res, 2013. 97(3): p. 589-97.

37. Yan, J., D.J. Bare, J. DeSantiago, et al., JNK2, a Newly-ldentified SERCA2 Enhancer, Augments an Arrhythmic [Ca(2+)](SR) Leak-Load Relationship. Circ Res, 2021. 128(4): p. 455-470.

38. Liang, X., Q. Zhang, X. Wang, et al., Reactive oxygen species mediated oxidative stress links diabetes and atrial fibrillation. Mol Med Rep, 2018. 17(4): p. 4933-4940.

39. Moe, G.W., G. Laurent, L. Doumanovskaia, et al., Matrix metalloproteinase inhibition attenuates atrial remodeling and vulnerability to atrial fibrillation in a canine model of heart failure. J Card Fail, 2008. 14(9): p. 768-76.

40. Lewkowicz, J., M. Knapp, A. Tankiewicz-Kwedlo, et al., MMP-9 in atrial remodeling in patients with atrial fibrillation. Ann Cardiol Angeiol (Paris), 2015. 64(4): p. 285-91.

41. Iwamoto, R. and E. Mekada, ErbB and HB-EGF signaling in heart development and function. Cell Struct Funct, 2006. 31(1): p. 1-14.

42. Munk, M., A.A. Memon, J.P. Goetze, et al., Hypoxia changes the expression of the epidermal growth factor (EGF) system in human hearts and cultured cardiomyocytes. PLoS One, 2012. 7(7): p. e40243.

43. Büttner, P., S. Werner, P. Sommer, et al., EGF (Epidermal Growth Factor) Receptor Ligands in Atrial Fibrillation: From Genomic Evidence to the Identification of New Players. Circ Arrhythm Electrophysiol, 2019. 12(4): p. e007212.

44. Li, X., L. Gao, Z. Wang, et al., Lipid profile and incidence of atrial fibrillation: A prospective cohort study in China. Clin Cardiol, 2018. 41(3): p. 314-320.

45. Magnussen, C., T.J. Niiranen, F.M. Ojeda, et al., Sex Differences and Similarities in Atrial Fibrillation Epidemiology, Risk Factors, and Mortality in Community Cohorts: Results From the BiomarCaRE 
Consortium (Biomarker for Cardiovascular Risk Assessment in Europe). Circulation, 2017. 136(17): p. 1588-1597.

46. Lopez, F.L., S.K. Agarwal, R.F. Maclehose, et al., Blood lipid levels, lipid-lowering medications, and the incidence of atrial fibrillation: the atherosclerosis risk in communities study. Circ Arrhythm Electrophysiol, 2012. 5(1): p. 155-62.

47. Huxley, R.R., K.B. Filion, S. Konety, and A. Alonso, Meta-analysis of cohort and case-control studies of type 2 diabetes mellitus and risk of atrial fibrillation. Am J Cardiol, 2011. 108(1): p. 56-62.

48. Begieneman, M.P., L. Rijvers, B. Kubat, et al., Atrial fibrillation coincides with the advanced glycation end product $N(\varepsilon)$-(carboxymethyl)lysine in the atrium. Am J Pathol, 2015. 185(8): p. 2096-104.

49. Raposeiras-Roubín, S., B.K. Rodiño-Janeiro, L. Grigorian-Shamagian, et al., Evidence for a role of advanced glycation end products in atrial fibrillation. Int J Cardiol, 2012. 157(3): p. 397-402.

50. Fujisawa, K., N. Katakami, H. Kaneto, et al., Circulating soluble RAGE as a predictive biomarker of cardiovascular event risk in patients with type 2 diabetes. Atherosclerosis, 2013. 227(2): p. 425-8.

51. Li, D., K. Shinagawa, L. Pang, et al., Effects of angiotensin-converting enzyme inhibition on the development of the atrial fibrillation substrate in dogs with ventricular tachypacing-induced congestive heart failure. Circulation, 2001. 104(21): p. 2608-14.

52. Aschar-Sobbi, R., F. Izaddoustdar, A.S. Korogyi, et al., Increased atrial arrhythmia susceptibility induced by intense endurance exercise in mice requires TNFa. Nat Commun, 2015. 6: p. 6018.

53. Valente, A.J., T. Yoshida, J.D. Gardner, et al., Interleukin-17A stimulates cardiac fibroblast proliferation and migration via negative regulation of the dual-specificity phosphatase MKP-1/DUSP-1. Cell Signal, 2012. 24(2): p. 560-568.

54. Onishi, R.M. and S.L. Gaffen, Interleukin-17 and its target genes: mechanisms of interleukin-17 function in disease. Immunology, 2010. 129(3): p. 311-21.

55. Yue, H., W. Liang, J. Gu, et al., Comparative transcriptome analysis to elucidate the therapeutic mechanism of colchicine against atrial fibrillation. Biomed Pharmacother, 2019. 119: p. 109422.

\section{Tables}

\section{Table 1 Key target information table of Que in the treatment of AF}




\begin{tabular}{llll} 
Uniprot ID & Gene name & Protein name & Degree \\
\hline P05231 & IL-6 & Interleukin-6 & 47 \\
\hline P15692 & VEGFA & Vascular endothelial growth factor A & 45 \\
\hline P05412 & JUN & Transcription factor AP-1 & 41 \\
\hline P00533 & EGFR & Epidermal growth factor receptor & 38 \\
\hline P14780 & MMP9 & Mitogen-activated protein kinase 1 & 38 \\
\hline P24385 & CCND1 & G1/S-specific cyclin-D1 & 38 \\
\hline P10145 & CXCL8 & Interleukin-8 & 38 \\
\hline P35354 & PTGS2 & Prostaglandin G/H synthase 2 & 37 \\
\hline P03372 & ESR1 & Estrogen receptor & 35 \\
\hline P01106 & MYC & Myc proto-oncogene protein & 35
\end{tabular}

Table 2 KEGG pathway enrichment analysis of Que against AF 
AGE-RAGE

signaling pathway

in diabetic

complications

Lipid and

atherosclerosis
29.23

35.38

Fluid shear stress

and

atherosclerosis

Proteoglycans in

cancer

26.15

24.61

Human

cytomegalovirus

infection

Kaposi sarcoma- $\quad 21.54$

associated

herpesvirus

infection

Hepatitis B

20.00

24.62

MAPK signaling

pathway

Salmonella

infection

21.54

20.00

Chemical

carcinogenesis -

receptor activation
7.01E- NOX4, VEGFA, CCND1, BAX, JUN, IL-6, PRKCA,

22 STAT1, IL-1B, SELE, CSCL8, TGFB1, THBD,

SERPINE, COLA1A, COL3A1, JAK2, MAPK14, CD42

1.45E- PTK2, MMP9, CAMK2B, PPARG, FOS, BAX, 23

20 JUN, IL6, NFKBIA, PRKCA, IL1B, SELE, CXCL8, NFE2L2, CXCL2, CASP1, JAK2, MAPK14, HSP90AA1, CCL5, RHOA, CDC42, HSPA42

1.51E- PTK2, MMP9, VEGFA, FOS, JUN, IL1B, SELE,

13 THBD, NFE2L2, NQ01, MAPK14, HSP90AA1, RHOA, GST01, ACTB

2.33E- PTK2, MMP9, CAMK2B, VEGFA, CND1, 13 PRKCA, MYC, TGFB1, COL1A1, FGFR1, ESR1, PRKACA, MAPK14, RHOA, CDC42, ACTB

PTK2, PTGS2, VEGFA, CCND1, BAX, IL6,

NFKBIA, PRKCA, MYC, IL1B, CXCL8, PRKACA,

MAPK14, CCL5, RHOA

2.64E- VEGFA, CCND1, FOS, BAX, JUN, IL6, NFKBIA,

STAT1, MYC, CXCL8, CXCL2, JAK2, MAPK14

3.34E- FOS, BAX, JUN, IL6, NFKBIA, PRKCA, STAT1, MYC, CXCL8, TGFB1, JAK2, MAPK14

7.75E- MAPT, INSR, VEGFA, FOS, JUN, PRKCA, MYC, IL1B, HSPB1, TGFB1, FGFR1, PRKACA, MAPK14, CDC42, HSPA2

6.95E- BAX, JUN, IL6, NFKBIA, MYC, IL1B, CXCL8, 14 CASP1, MAPK14, HSP90AA1, RHOA, CDC42, ACTB

9.16E- VEGFA, CCND1, FOS, JUN, PRKCA, MYC, 09

PPARA, JAK2, ESR1, PRKACA, HSP90AA1, GSTO1

Table 3 Molecular docking results 


\begin{tabular}{|llll|}
\hline Compound & Target & PDB & Energy $(\mathrm{kcal} / \mathrm{mol})$ \\
\hline Quercetin & IL-6 & $409 \mathrm{H}$ & -4.7 \\
\hline Quercetin & VEGFA & $4 \mathrm{G} 1 \mathrm{~W}$ & -6.47 \\
\hline Quercetin & JUN & 1JUN & -3.64 \\
\hline Quercetin & MMP9 & 5TH6 & -5.15 \\
\hline Quercetin & EGFR & 3IKA & -5.25 \\
\hline
\end{tabular}

Figures 


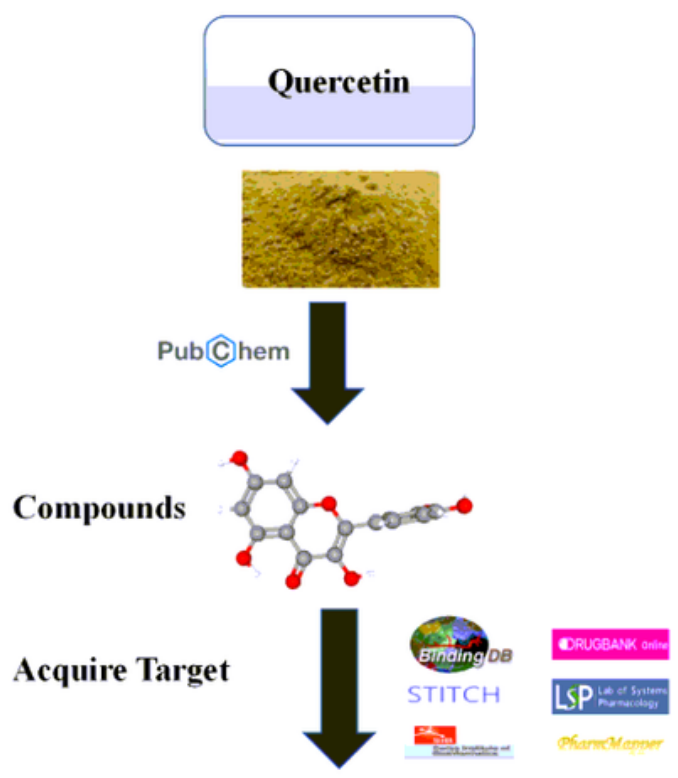

Get quercetin targets

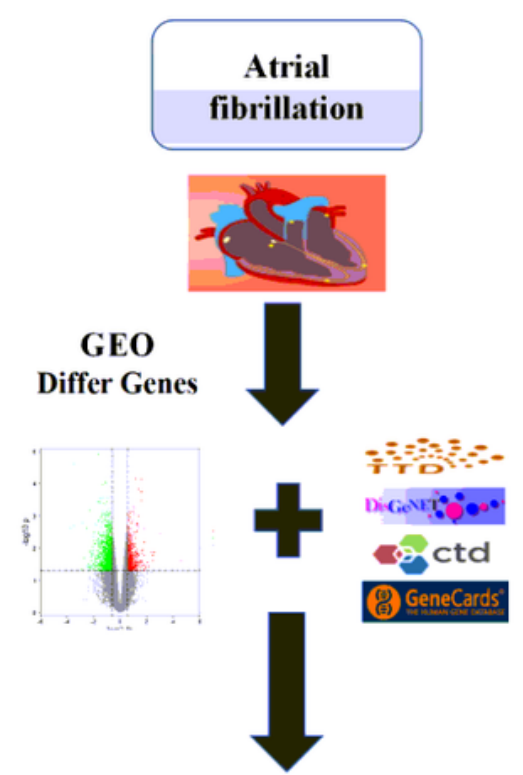

Get AF disease genes

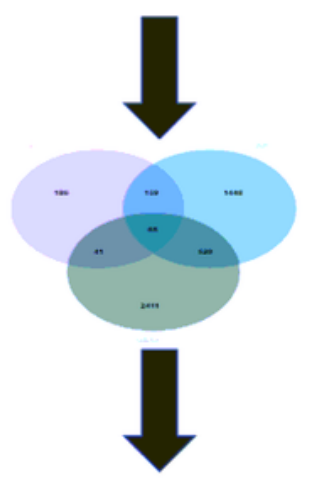

PPI Network analysi

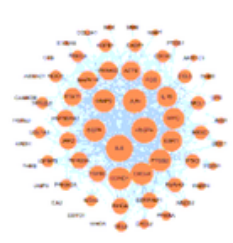

GO and KEGG enrichment analysis

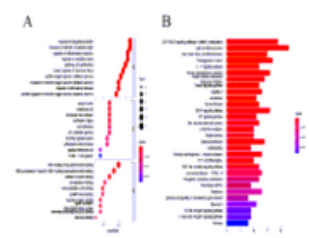

Que targetmajor pathwayAF

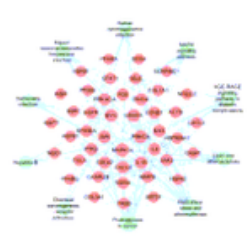

MAPK signaling pathway upregulation targets

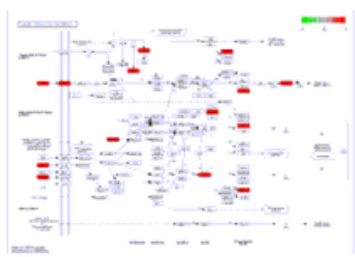

Molecular docking and differential analysis of core targets

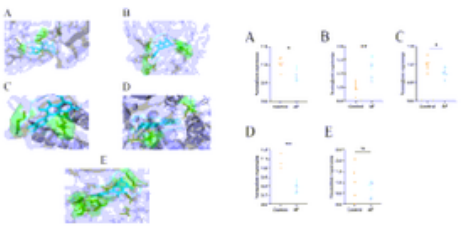

\section{Figure 1}

Graphical abstract 
A

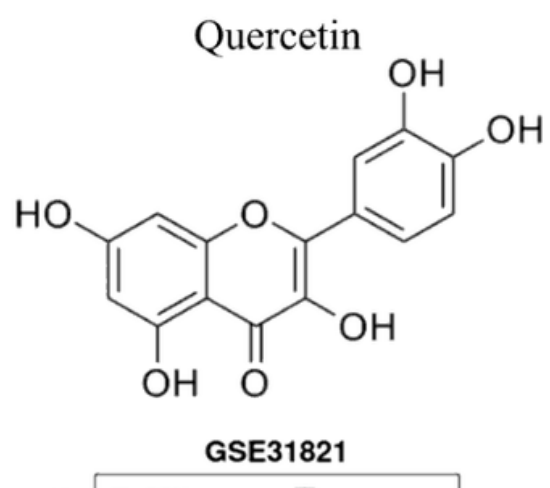

C

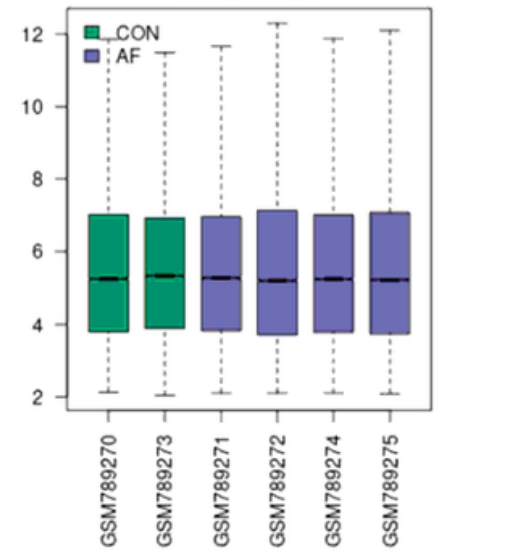

D

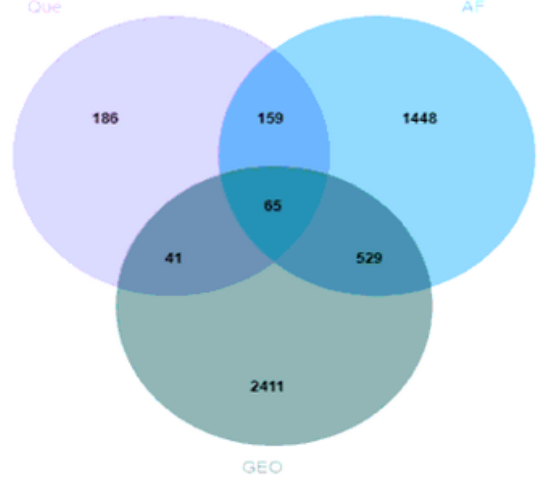

B

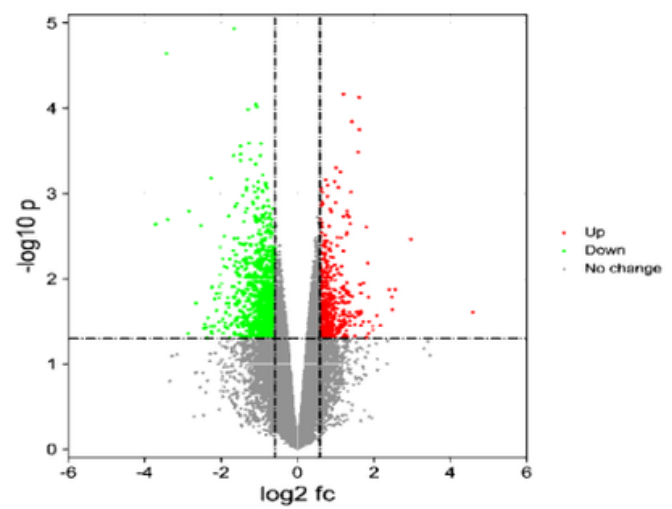

E

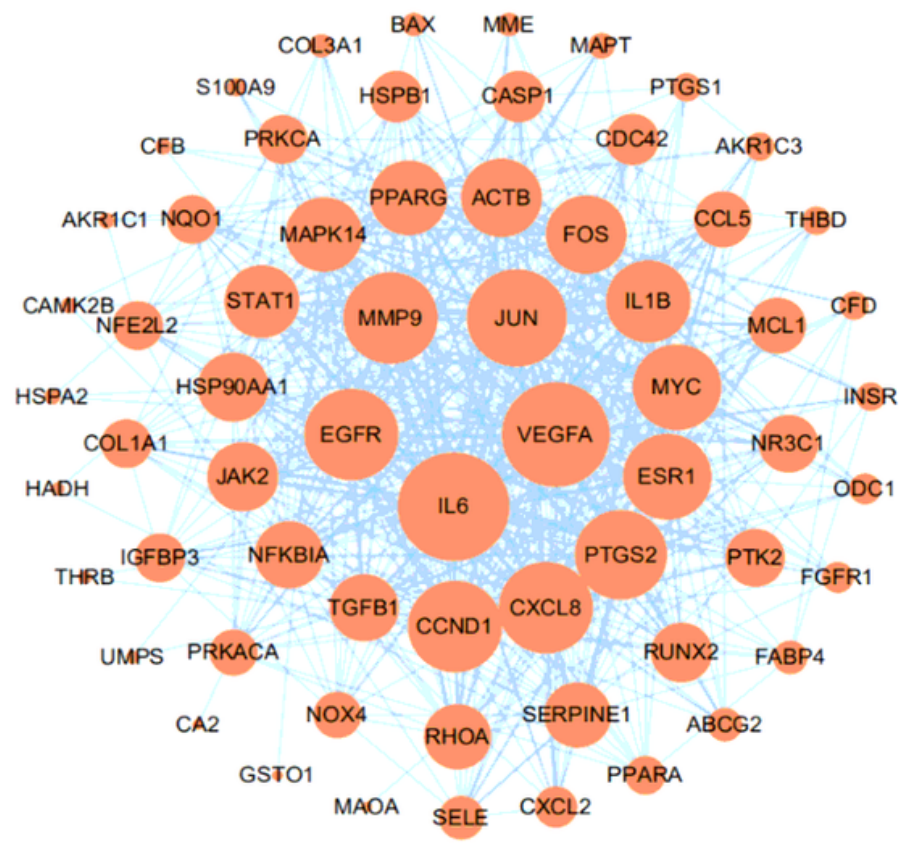

Figure 2

Protein-protein interaction (PPI) networks construction for target proteins of Que against AF. (A) The chemical structure of the quercetin. (B)GEO Volcano Map. Red upregulates the target, green downregulates the target; (C) GEO Difference Chart Differential. Gene expression in normal subjects (green) vs AF patients (purple). (D)Venn diagram of Que AF and GEO. (E)PPI network of Que against AF. The larger the circle and the darker the color, the stronger the correlation with the therapeutic target. 
A

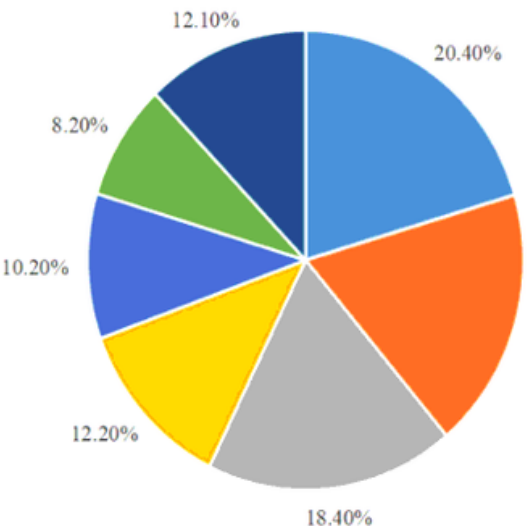

$18.40 \%$

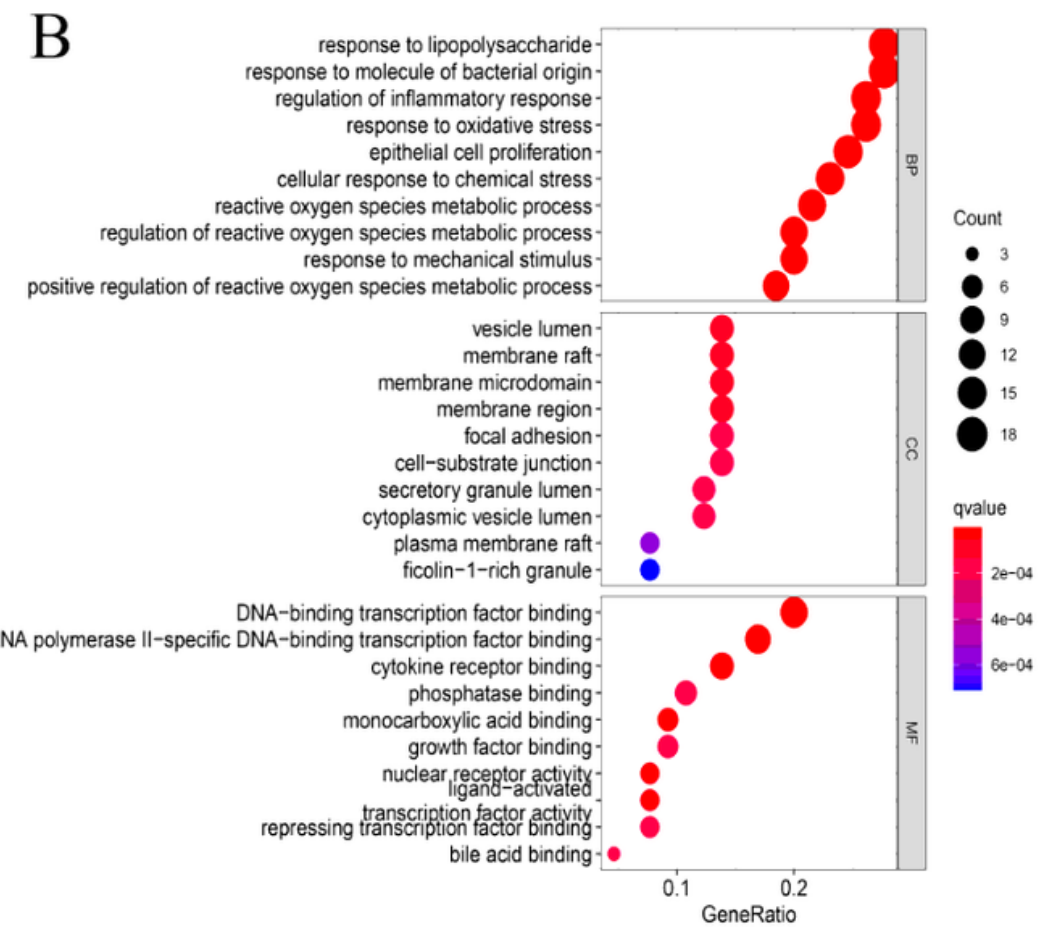

C

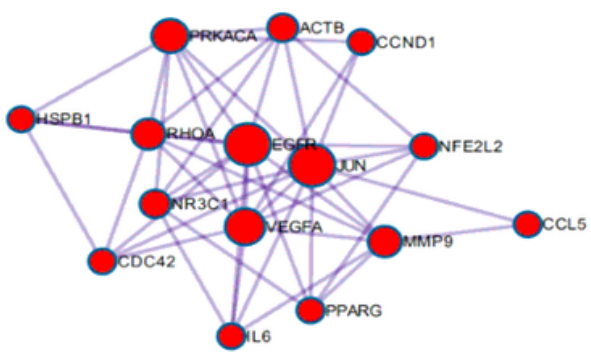

Mcode_1 GO:0048660

Regulation of smooth muscle cell proliferation

D

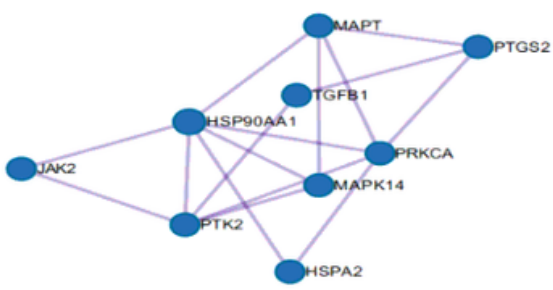

Mcode_2 GO:0042307

Positive regulation of protein import into nucleus

$\mathrm{E}$

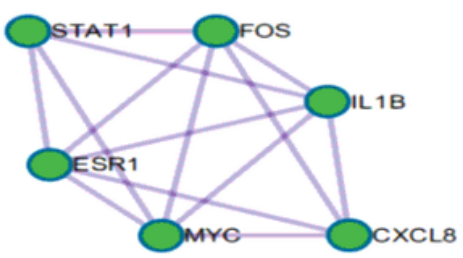

Mcode_3 GO:1902532

Negative regulation of intracellular signal transduction

\section{Figure 3}

Bioinformatics analysis of target proteins of Que against AF. (A) Panther classification categorized target proteins of Que against AF. The figures next to the pie chart indicate the percentage of protein in the given functional class. (B) GO, BP, CC and MF enrichment analysis of interselection targets. The intensity of the color represents the adjusted $p$ value, and the bubble size corresponds to the number of genes. $(C, D, E)$ The Molecular Complex Detection (MCODE) algorithm has been used to identify densely connected network components. 
A
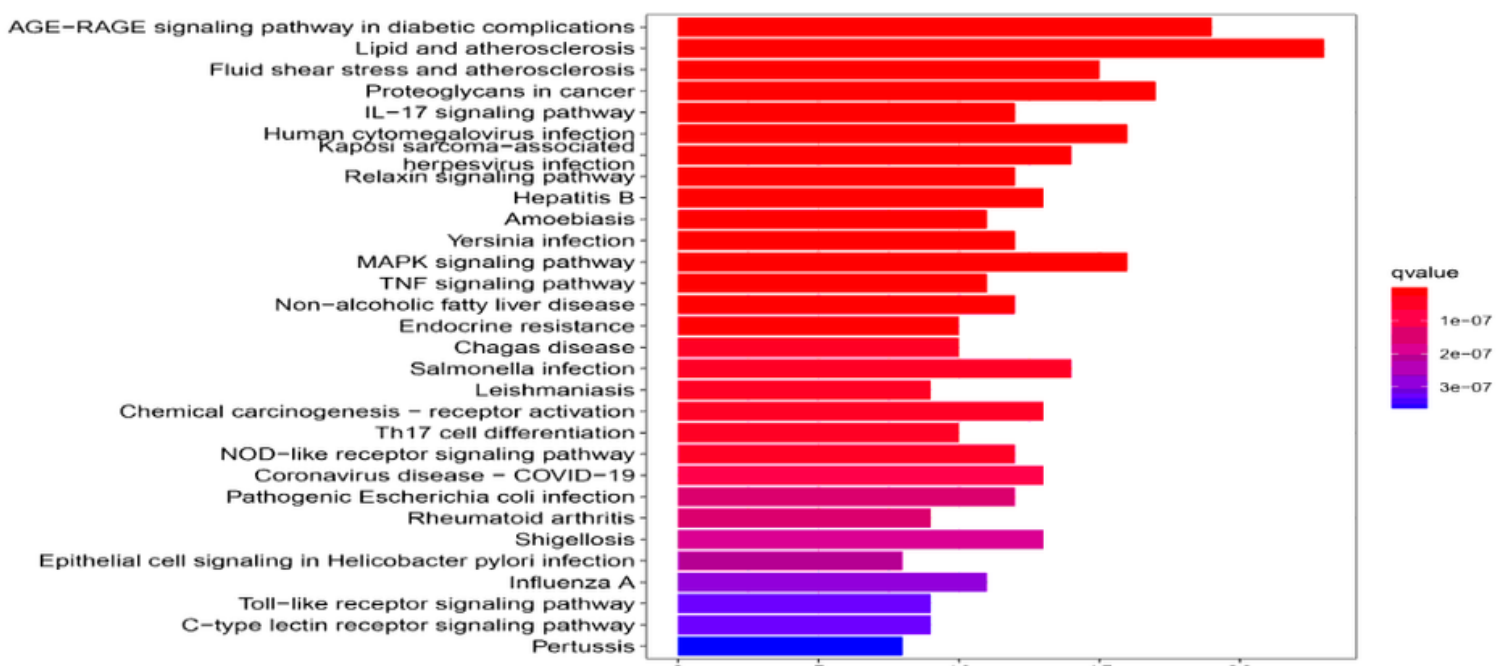

B
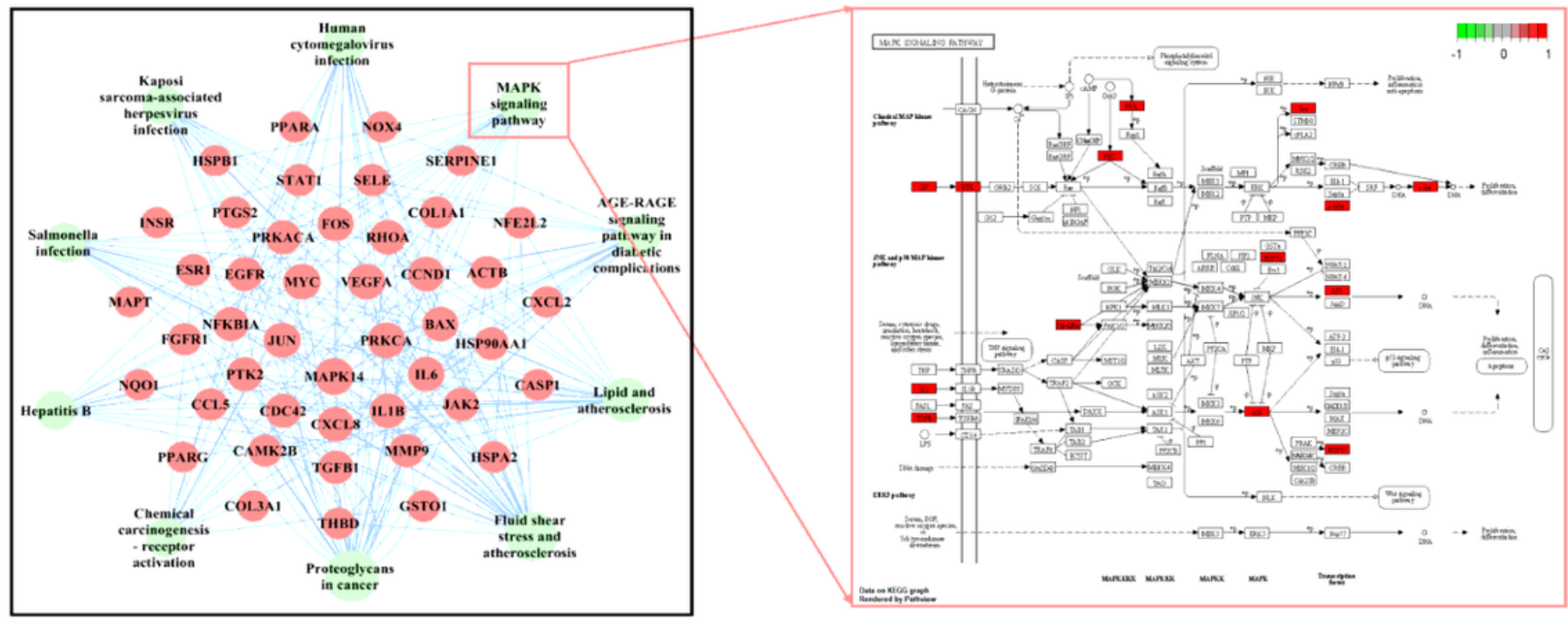

\section{Figure 4}

KEGG pathway enrichment analysis(A) KEGG enrichment analysis of interselection targets. The intensity of the color represents the adjusted $p$ value. (B) Que target-major pathway-AF. The middle purple circle is the relevant pathway and the outer circle is the relevant target in the pathway. (C) MAPK detailed pathway map. The red area is the MAPK upregulation target of the key pathway of AF for Que treatment. 
A

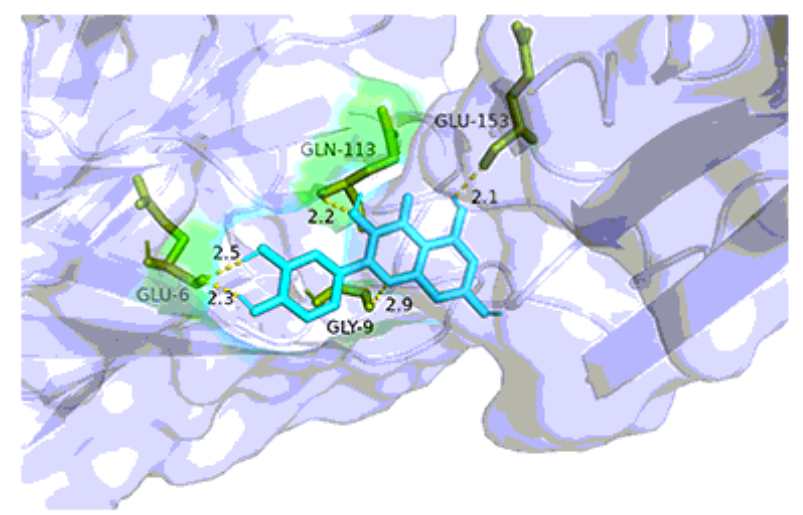

$\mathrm{C}$

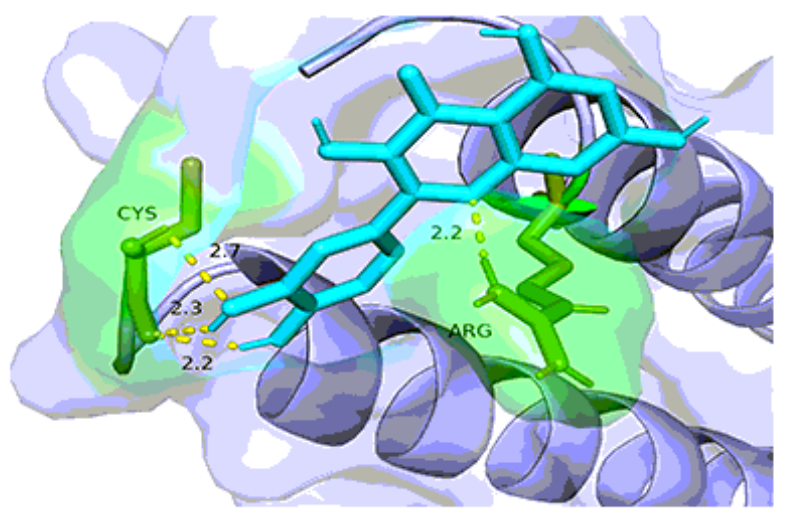

B

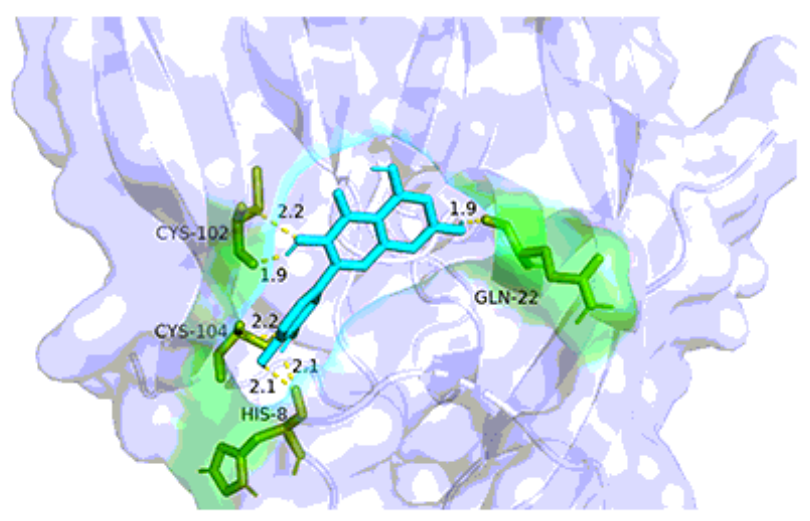

$\mathrm{D}$

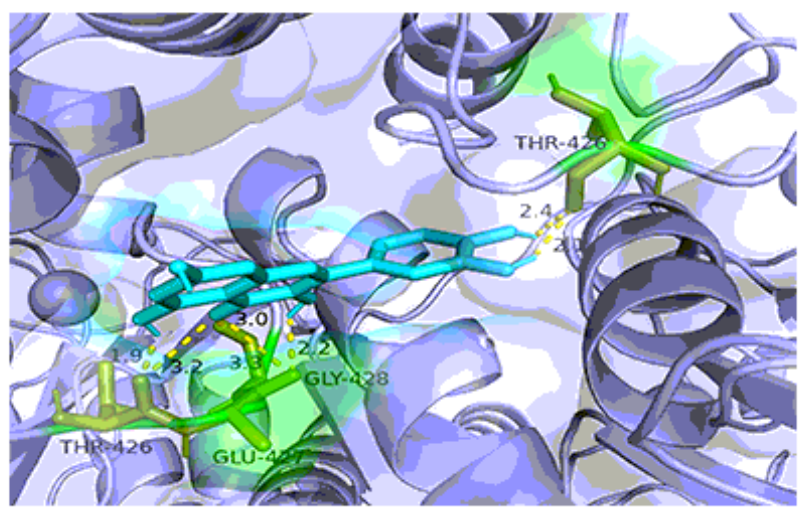

$\mathrm{E}$

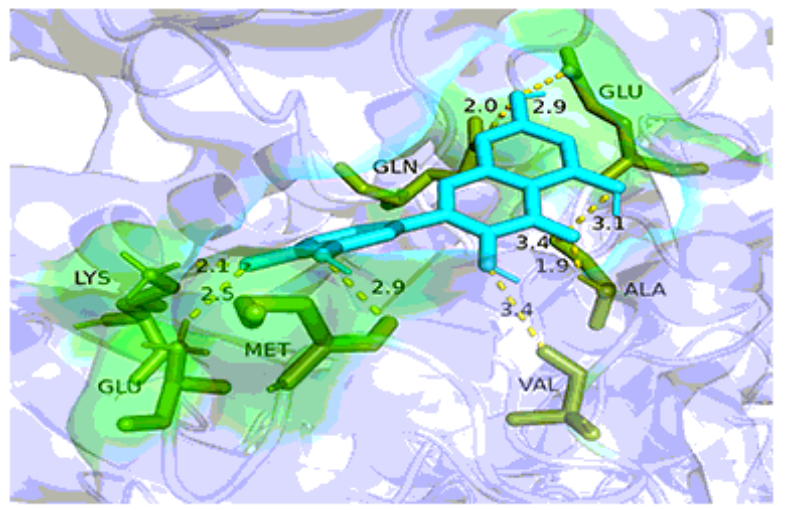

Figure 5

Docking pattern of Que with key target molecules. (A) IL-6 and Que molecular docking; (B) VEGFA and Que molecular docking; (C) JUN and Que molecular docking; (D) MMP9 and Que molecular docking; (E) EGFR and Que molecular docking. 


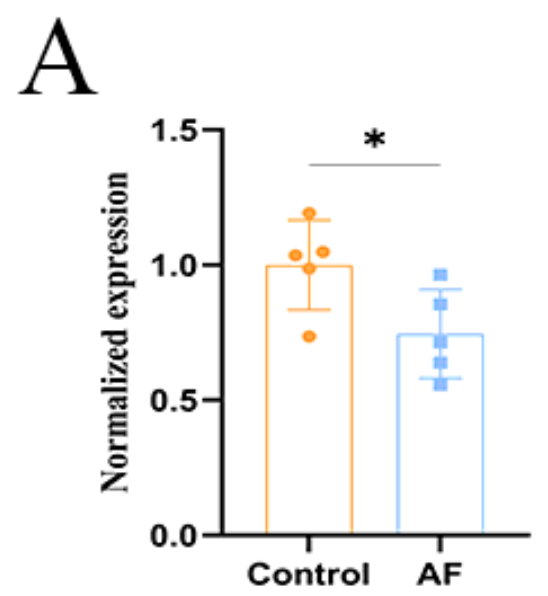

$\mathrm{B}$
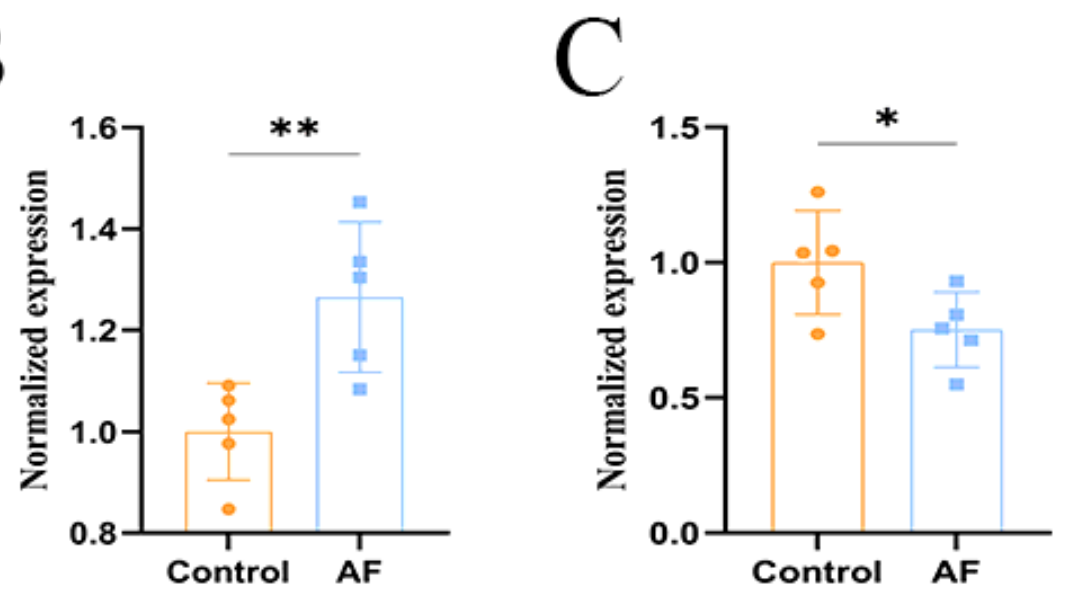

$\mathrm{D}$

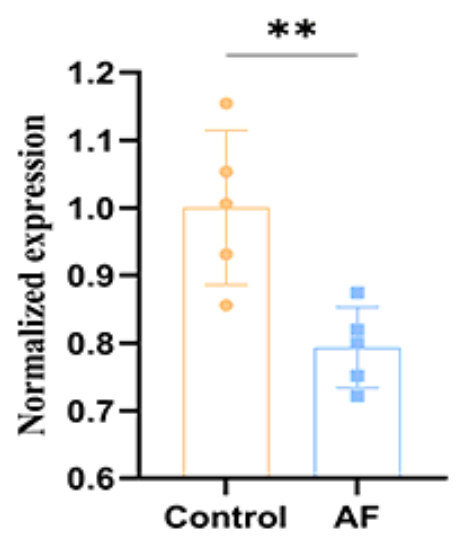

$\mathrm{E}$

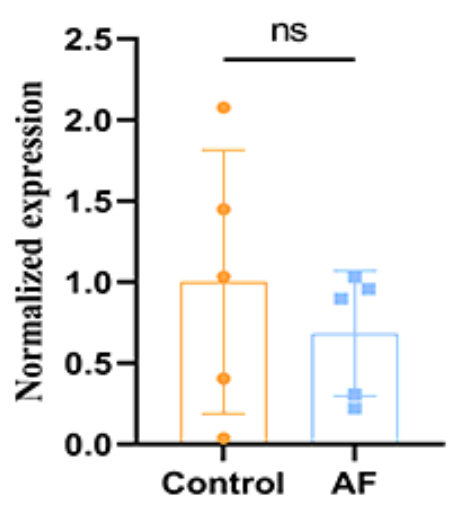

Figure 6

The core action targets of Que on AF were differentially expressed in the GEO data set. Left atrial healthy controls $n=5$, AF group $n=5$. Values are expressed as mean \pm SD. Compared with Control, ${ }^{*}<0.05$ and $\star * P<0.01$. (A) IL-6 differential expression; (B) VEGFA differential expression; (C) JUN differential expression; (D) EGFR differential expression; (E) MMP9 differential expression. 